\title{
Children Tooth Brushing Behavior and Oral Microbiota: A Pilot Study
}

\author{
Susana J. Calderon ${ }^{1, *}$, Seon Yoon Chung ${ }^{1}$, Christopher J. Fields ${ }^{2}\left(\mathbb{D}\right.$ and Nathan T. Mortimer ${ }^{3}(\mathbb{C}$ \\ 1 Mennonite College of Nursing, Illinois State University, Normal, IL 61790, USA; schung6@ilstu.edu \\ 2 High Performance Computing in Biology Group, Roy J. Carver Biotechnology Center, University of Illinois \\ Urbana-Champaign, Champaign, IL 61801, USA; cjfields@illinois.edu \\ 3 School of Biological Sciences, Illinois State University, Normal, IL 61790, USA; ntmorti@ilstu.edu \\ * Correspondence: sjcalde@ilstu.edu; Tel.: +1-(309)-438-2562
}

check for updates

Citation: Calderon, S.J.; Chung, S.Y.; Fields, C.J.; Mortimer, N.T. Children Tooth Brushing Behavior and Oral Microbiota: A Pilot Study. Oral 2021, 1, 112-121. https://doi.org/10.3390/ oral1020012

Received: 10 March 2021

Accepted: 5 May 2021

Published: 14 May 2021

Publisher's Note: MDPI stays neutral with regard to jurisdictional claims in published maps and institutional affiliations.

Copyright: (c) 2021 by the authors. Licensee MDPI, Basel, Switzerland. This article is an open access article distributed under the terms and conditions of the Creative Commons Attribution (CC BY) license (https:/ / creativecommons.org/licenses/by/ $4.0 /)$.

\begin{abstract}
By age 17, almost $80 \%$ of US children have dental caries resulting from cariogenic bacteria that could be reduced with tooth brushing. This cross-sectional, correlational pilot study aims to understand the association between tooth brushing and oral microbiota in children. Oral specimens and survey data were collected from a convenience sample of 16 children, aged 7-12, and attending a community dental clinic. Descriptive statistics were used to analyze sample characteristics and tooth brushing behavior. Biospecimens were analyzed using Polymerase Chain Reaction (PCR) amplification and $16 \mathrm{~S}$ ribosomal RNA (rRNA) gene sequencing. Beta diversity measures were compared across tooth brushing groups using Permutational Analysis of Variance (PERMANOVA) and Analysis of Similarities (ANOSIM) tests in R. The frequency of tooth brushing in this sample was once a day (brush1; 43.8\%), twice a day (brush2; $43.8 \%$ ), or more than twice a day (12.6\%). Major phyla found in the subjects' upper and lower teeth were the following: Proteobacteria, Fusobacteria, Firmicutes, Bacteroidetes, and Actinobacteria. The relative abundance of Actinobacteria was significantly lower in brush2 as compared to brush1 $(p=0.001)$, whereas the relative abundance of Proteobacteria was significantly higher $(p=0.025)$. The association between tooth brushing frequency and microbiome beta diversity was significant ( $p=0.005$ by PERMANOVA and $p=0.002$ by ANOSIM). This study demonstrates that tooth brushing frequency could affect the proportional composition of the oral microflora. Additional research on the implication of these changes is warranted.
\end{abstract}

Keywords: children; tooth brushing; oral microbiota

\section{Introduction}

Dental caries remains as one of the most prevalent childhood diseases, and it causes problems well beyond the teeth: it is associated with cardiovascular disease, obesity, and lifelong systemic diseases such as Type II Diabetes Mellitus [1]. Despite prevention efforts, $17.4 \%$ of children under 9 years old have at least one cavity or filling in their primary teeth [2], and by age 17 the rate rises to $78 \%$ [3]. Yearly, thousands of children of different ages have dental procedures in surgical suites due to dental problems [3]. Dental treatment and procedures cost industrialized countries billions of dollars [4]. Previous researchers have provided important insights into the influence of oral health behavior, especially the lack of tooth brushing by children and the association of not only caries but other chronic conditions such as Type II Diabetes Mellitus. One of the most inexpensive prevention measures is tooth brushing, which prevents caries and common chronic conditions in adulthood. Tooth brushing frequency in children offers benefits towards preventing caries and decreasing the expended costs and resources in countries around the world [5].

While recent studies have suggested the beneficial roles of the human microbiome in overall health, the role of the oral microbiome in promoting oral health and decreasing systemic inflammation is understudied [6]. Recently, associations among oral health, the oral microbiota, and systemic diseases have been postulated [7]. Both biological and 
environmental factors shape the profile of the oral microbiome in children: they acquire their oral microbiomes at birth from their mothers, and interaction with their environment results in commensal bacteria of more than 1000 taxa [7]. The role of different commensal bacteria in causing caries remains unclear, and although the oral health behaviors of tooth brushing — using fluoride toothpaste, dental flossing, and using mouthwash—are known to improve oral health, little is known about the influence of the frequency of tooth brushing on the oral microbiome. A low $\mathrm{pH}$ level may create an acidic environment too low for some caries-producing bacteria such as Streptococcus mutans [8]. Reducing the biofilm produced by the bacteria, which demineralizes the tooth enamel, results in a decrease of caries risk. Moreover, tooth brushing may reduce the abundance of caries-related bacterial phyla $[9,10]$. The purpose of this pilot study was to understand the association between the frequency of tooth brushing and the oral microbiota in children.

\section{Materials and Methods}

A pilot study using a cross-sectional, correlational design was conducted to observe the associations between oral health behaviors, in particular tooth brushing, and oral microbiota. A convenience sample of sixteen children aged 7-12 years old, from diverse socioeconomic levels and ethnic backgrounds, was recruited from a Midwest community dental clinic. The inclusion criteria were children (a) 7-12 years old, (b) with a parent/guardian who was able to provide consent and child assent, and (c) who were able to communicate in oral and written English. Children who had been on antibiotics, oral inhalers, steroids, or Non-steroidal anti-inflammatory drugs (NSAIDs) in the previous 3 months were excluded. The recruitment protocol focused on children not taking oral medication for acute or chronic conditions at that time in order to avoid disturbance in the ecological environment of the oral microbiome. Based on audits in pertinent literature, the sample size was calculated using moderate effect size, statistical power of $80 \%$, and an alpha of 0.05 , with sample size $n=10$ [11]. This study was approved by the Illinois State University Institutional Review Board (IRB-2018-45).

\subsection{Data Collection}

We administered two types of surveys. A demographic questionnaire, filled out by the parent and the child, collected information on age, race, ethnicity, gender, if living with parents or others, education, and parent's employment. An oral health behavior questionnaire, written in language suitable for children, was used to collect data directly from the children about their tooth brushing frequency, dental flossing, and use of mouth wash and fluoride toothpaste. The survey had a Cronbach's $\alpha=0.70$, moderate internal consistency. The children completed the oral health questionnaire prior to having their saliva sampled. We also surveyed dental records for variables on history of caries, history of prior disease, and history of prior medications.

Before their dental examination, we collected samples of their oral microbiota using sterile swabs from the upper and lower teeth, the right and left cheeks, the upper palate, and the tongue. This study used specimens from the upper and lower teeth, collected from the following specific surface sites: posterior, anterior, molars, incisors, and premolars. We used the aseptic technique to collect the specimens, following the manufacturer's instructions (sample collection quick protocol, Zymo Research). We began by wearing gloves and opening the package containing the swab and collection tube. We instructed the participant to open their mouth, swabbed each sample site with an individual package, swabbing several times with a back-and-forth motion before inserting the swab in the collection tube. After acquiring each individual sample, we inserted the swab in the collection tube, broke off the tip, closed the collection tube, and inverted it several times to mix the prefilled Zymo Research DNA/RNA shield. Each oral sample was collected and deposited separately using a Zymo Research DNA/RNA shield collection tube with swab, according to the manufacturer's instructions. Each collection tube was prefilled with $1 \mathrm{~mL}$ of DNA/RNA shield to preserve the nucleic acid content of the samples. The oral samples 
were collected from all children at the same time, the same day of the week, and only once. Oral samples were deidentified and labeled with a serial number designation to coordinate with the oral health questionnaire. The oral samples were stored at $-20^{\circ} \mathrm{C}$, following the manufacturer's instructions until analysis. From the 96 total oral samples collected, the 32 samples from upper and lower teeth were used for analysis.

\subsection{Data Analyses}

Descriptive statistics were used to analyze sample characteristics and oral health behavior, particularly tooth brushing frequency, using IBM SSPS statistics 24 version. Polymerase Chain Reaction (PCR) amplification and 16S rRNA gene sequencing were used to analyze oral specimens. Differences in oral microbial composition across tooth brushing groups were tested using Permutational Analysis of Variance (PERMANOVA) and Analysis of similarities (ANOSIM) in R.

\subsection{Microbiome Analysis and Sequence Data Processing}

DNA extraction and 16S rRNA Next generation sequencing. DNA extraction and isolation were completed following the ZymoBIOMICS DNA Microprep Kit manufacturer's instructions. Extracted DNA samples were submitted to the Functional Genomics Unit and the High-Performance Biological Computing Unit at the University of Illinois for amplification using universal V4 Primer TACGGTAGCAGAGACTTGGTCTGACTACHVGGGTATCTAATCC. PCR-amplified products and labels for 16S rRNA were then sequenced using Fluidigm and Illumina MiSeq sequencing technology, following standard protocols.

The oral microbiomes of participants were analyzed in depth using 16S rRNA gene sequencing. This approach allowed us to identify bacteria to the level of phylum in little time and with low cost $[12,13]$.

Workflow. Raw FASTQ data from the samples were processed using a Nextflowbased workflow wrapping dada2, release 1.6.0 [14] and R v3.4.2 [15]; the workflow was configured and deployed on the Biocluster HPC cluster at the University of Illinois (http: / / biocluster.igb.illinois.edu (accessed on 25 November 2018)). The workflow was configured with the use of dada2 to trim, dereplicate, and denoise amplicon sequence data; Silva to generate a count table of amplicon sequence variants (ASV's) and a taxonomic assignment of the resulting ASV's [16]; DECIPHER 2.6.0 to align the ASV sequences in multiple sequences [17]; and FastTree v2.1.10 to analyze the maximum likelihood phylogeny [18]. We focused on the V4 region analysis for the samples and executed the dada2 pipeline.

Assigning taxonomy. The Silva v32 database was utilized for taxonomic assignment within dada2 using their implementation of the RDP Classifier [19]. Taxonomic rank assignment was additionally performed on the V4 data set. The snapshot of the workflow used for this analysis can be found here: https: / github.com/HPCBio/16S-rDNA-dada2 -pipeline/releases/tag/2018-calderon (accessed on 25 November 2018). All data analyses were performed using R v.3.5.1, and the packages phyloseq v. 1.26 [20] and vegan v2.5 [14].

Decontaminating data. Four replicate samples were removed. Data were further filtered to remove potential contaminants with the R decontam v1.2 package [21], using the water sample as a control; further filtering removed ASVs likely originating from mitochondria (eukaryotic origin). After these steps, samples with zero counts remaining were removed. 16S rRNA sequencing results were obtained from all 32 oral samples. Following quality control filtering for contaminating sequences and read number, two samples were eliminated, leaving 30 for further analysis.

\subsection{Statistical Analysis}

Alpha and beta diversity analysis. Operational Taxonomic Units (OTUs) were identified from sequencing data using phyloseq [20], and the abundance of identified phyla. Alpha diversity analyses were performed using phyloseq; a mixed-model analysis was performed using linear and nonlinear mixed effect model RPackage v3.4 [22] to correct for potential group effects (repeated measures). Beta diversity was assessed using phyloseq 
and vegan package in R; sample data was initially prevalence-filtered to remove any ASV's not found in at least two samples with a minimal CPM (count per million) of one. Counts were agglomerated to the genus level, retaining ASV's where the genus was not assigned as 'NA'. Three Samples were removed as outliers based on low overall counts, beta diversity analysis, and anomalous microbiome frequency. Beta diversity measures were compared across tooth brushing groups using PERMANOVA and ANOSIM tests.

\section{Results}

\subsection{Descriptive Data}

Of the 16 children aged 7-12 years who participated in the study, a little less than a third were Hispanic (28.6\%), followed by Caucasian and African American (21.4\% each), and Asian and Bi-racial (14.3\% each). Two participants opted out from reporting race. More than half of the participants were male (62.5\%). The average age was 9 years old; half of the children were in 6 th grade and the other half in 2 nd or 4 th ( $25 \%$ each). Seventy-five percent of the participants had parents who were both working and earning an income, and 75\% of the household inhabitants had four people in their home (see Table 1). All participants had a history of caries in the past, $31.3 \%$ of the participants had a history of chronic disease (e.g., asthma), and $43.7 \%$ of the participants had taken oral medication in the previous year. Participants reported a high frequency of tooth brushing: $87 \%$ brushed either once or twice a day, and $6 \%$ brushed more than twice a day (see Table 2). The majority of participants (81.3\%) never flossed. More than half of the participants (56.3\%) used mouth wash once or twice daily, and the rest either never used mouthwash or used it once a week.

Table 1. Sample Demographics.

\begin{tabular}{|c|c|c|}
\hline Characteristics $(\mathrm{N}=16)$ & & Percentage/Mean (Range) \\
\hline \multirow{2}{*}{ Gender } & Male & $62.5 \%$ \\
\hline & Female & $37.5 \%$ \\
\hline \multicolumn{2}{|l|}{ Age } & 9.1 years (7-12 years) \\
\hline \multirow{3}{*}{ Grade } & Second & $25 \%$ \\
\hline & Fourth & $25 \%$ \\
\hline & Sixth & $50 \%$ \\
\hline \multirow{5}{*}{ Race } & Caucasian & $21.4 \%$ \\
\hline & African American & $21.4 \%$ \\
\hline & Hispanic & $28.6 \%$ \\
\hline & Asian & $14.3 \%$ \\
\hline & Bi-racial & $14.3 \%$ \\
\hline \multirow{2}{*}{ Household Occupancy } & Up to Four & $75 \%$ \\
\hline & Four to Eight & $25 \%$ \\
\hline \multirow[b]{2}{*}{$\begin{array}{l}\text { Parent working in } \\
\text { the household }\end{array}$} & Mother and Father & $75 \%$ \\
\hline & $\begin{array}{l}\text { Grandmother } \\
\text { and/or Grandfather }\end{array}$ & $25 \%$ \\
\hline \multirow{2}{*}{ History of Caries } & Yes & \multirow{2}{*}{$100 \%$} \\
\hline & No & \\
\hline \multirow{2}{*}{ Chronic disease } & Yes & $31.3 \%$ \\
\hline & No & $68.7 \%$ \\
\hline \multirow{2}{*}{ Oral Medication } & Yes & $43.7 \%$ \\
\hline & No & $56.3 \%$ \\
\hline
\end{tabular}


Table 2. Children Tooth brushing Behavior.

\begin{tabular}{ccl}
\hline Oral Health Behavior & Frequency & Percentage \\
\hline \multirow{3}{*}{ Tooth brushing } & Never & $6.3 \%$ \\
& Once a day & $43.8 \%$ \\
& Twice a day & $43.8 \%$ \\
& More than twice a day & $6.3 \%$ \\
\hline \multirow{2}{*}{ Flossing } & Never & $81.3 \%$ \\
& Once a day & $6.3 \%$ \\
& Twice a day & $6.3 \%$ \\
& More than twice a day & $6.1 \%$ \\
\hline \multirow{2}{*}{ Mouth Wash } & Never & $37.0 \%$ \\
& Once a day & $37.0 \%$ \\
& Twice a day & $19.3 \%$ \\
\hline
\end{tabular}

\subsection{Microbiome Analysis}

Six hundred and sixty taxa were identified in 30 oral specimens following the decontamination and quality control process. All of these taxa were identifiable at the level of phyla. The major phyla found in the upper and lower teeth of the subjects in the tooth brushing groups (brush1: once a day; brush2: twice a day) were the following: Proteobacteria, Fusobacteria, Firmicutes, Bacteroidetes, and Actinobacteria (Figure 1a). Five hundred and ninety-one taxa were identified to the genera level (Figure 1b).
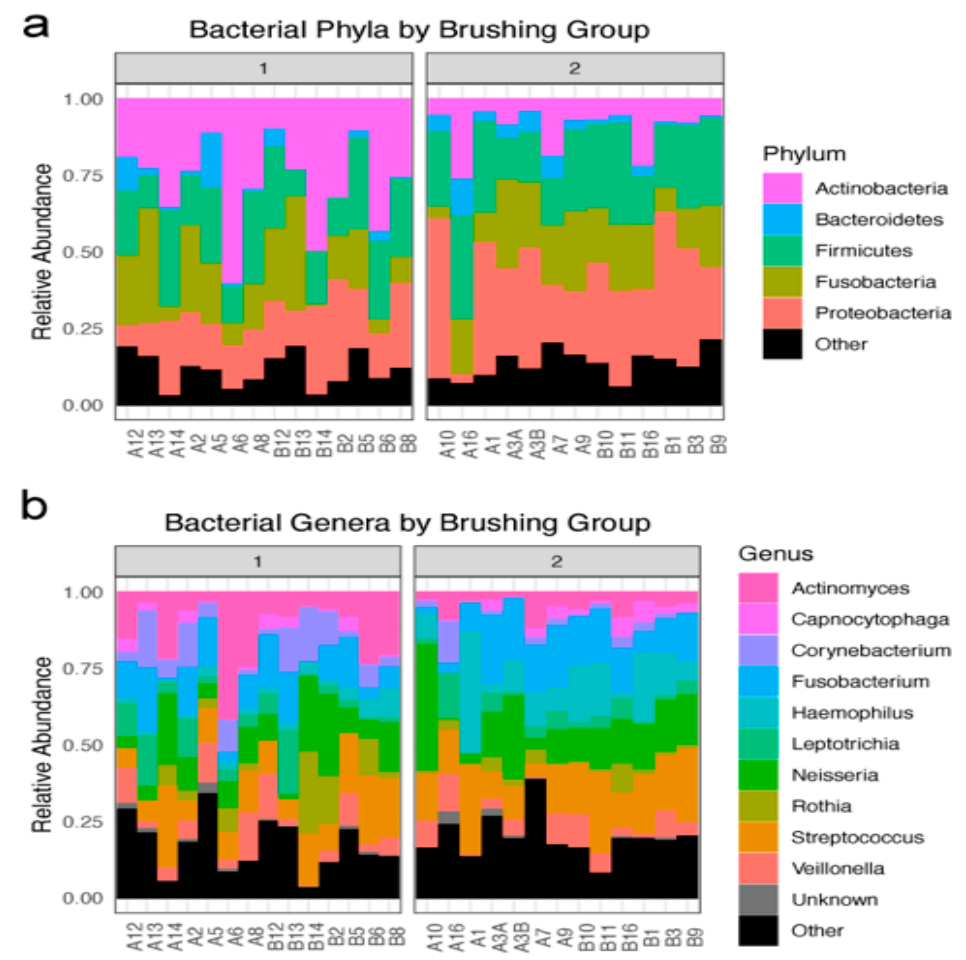

Figure 1. Overview of identified bacterial taxa: (a) Bacteria phyla by tooth brushing groups: brush1: once a day; brush2: twice a day. Relative abundance of the top five phyla, with an "other" group, in each participant sample within each tooth brushing group. (b) Bacterial genera by tooth brushing groups: brush1: once a day; brush2: twice a day. Relative abundance of the top 10 genera, with "other" and unknown groups, in each participant sample within each tooth brushing group.

Alpha diversity statistics were used to further characterize the samples. Alpha rarefaction curves were plotted for each subject in each tooth brushing group. The curves reached a plateau for each subject, suggesting that most of the diversity in the samples had 
been captured (Figure 2a). Four distinct alpha diversity measures (Observed ASV richness, Chao1 richness, Shannon index, and inverse Simpson index) were calculated to estimate within sample species richness. These measures revealed that there is variation between subjects, but that alpha diversity measures are not significantly impacted by tooth brushing status (Figure 2b).

\section{a}

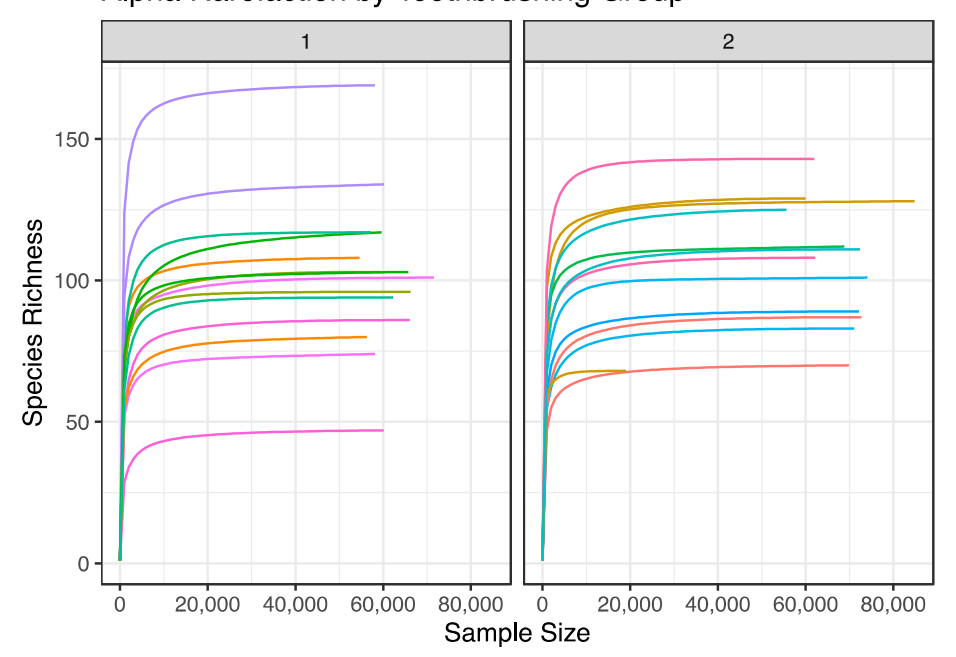

\section{Subject_ID}

$-1$

$-2$

$-3$

$-5$

$-6$

$-7$

$-8$

$-9$

$-10$

$-11$

$-12$

$-13$

- 14

b

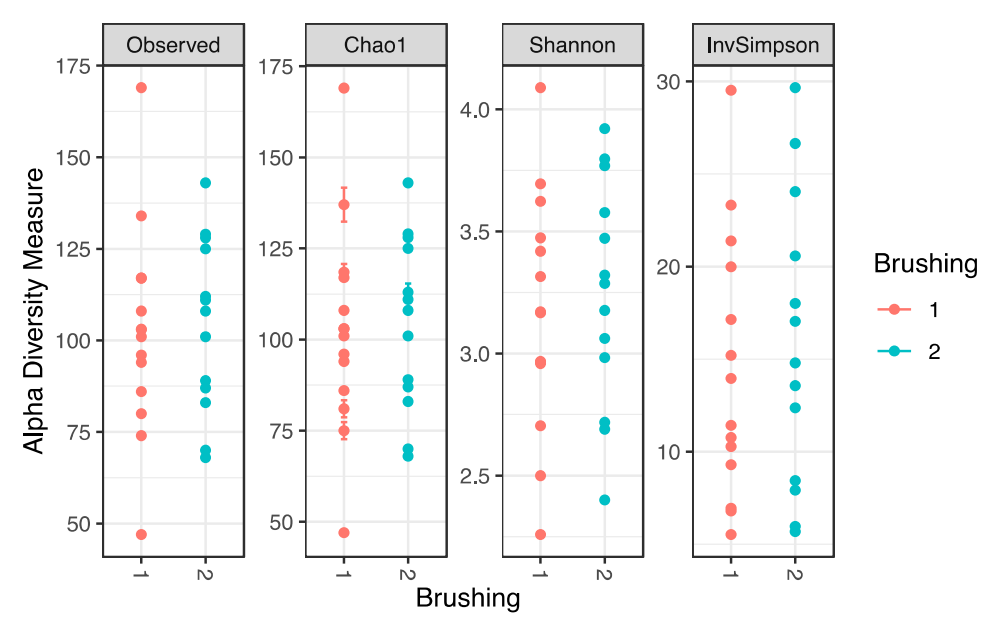

Figure 2. Sample alpha diversity: (a) Alpha Rarefaction by brushing group. Rarefaction curve for each subject. (b) Alpha diversity measures by brushing group. Abbreviations: Observed = Observed ASV; InvSimpson = inverse Simpson. Tooth brushing groups: brush1: once a day (red); brush2: twice a day (blue).

Beta diversity statistics were used to characterize differences between samples. Graphing the first two axes of a principal coordinates analysis (PCoA) of the weighted unifrac distances between subjects revealed a clustering of subjects into the two brushing groups, although there is some mixing of the two groups (Figure 3a). Hierarchical clustering by Ward's method 2 was used to further characterize the relationship between subjects. The resulting dendrogram agrees with the PCoA ordination plot; there is a split between the brushing groups, with a few of the brush2 group subjects found in the brush1 cluster (Figure 3b). 


\section{a}

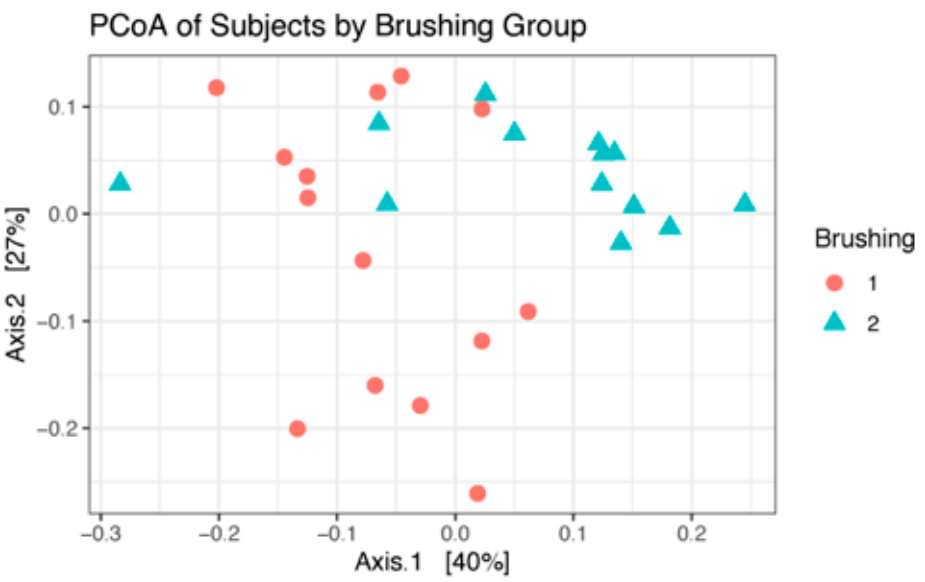

b Hierarchical Clustering by Brushing Group

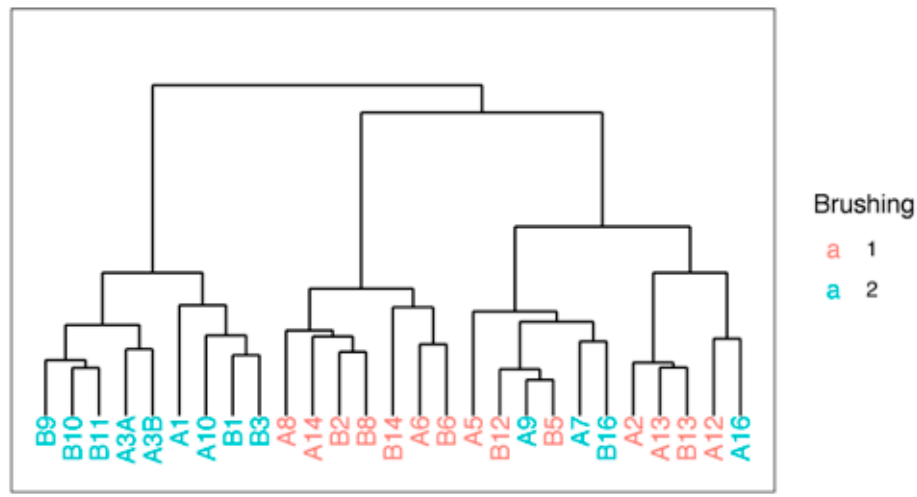

Figure 3. Beta diversity: (a) Ordination of the first two axes from principal coordinates analysis (PCoA) of subjects by brushing group. (b) Dendrogram of hierarchical clustering of subjects in each brushing group. Brushing groups: brush1: once a day (red); brush2: twice a day (blue).

\subsection{Correlation between Tooth Brushing Frequency and Microbiota}

Tooth brushing frequency was significantly associated with microbiome beta diversity, a measure of the difference in microbial community composition between samples. The dissimilarity between samples was calculated using the weighted UniFrac distance method and tested using PERMANOVA. The distance between brushing conditions was highly significant $\left(\mathrm{F}=4.49, \mathrm{R}^{2}=0.152, p=0.005,999\right.$ permutations). This result was further tested using the analysis of similarities (ANOSIM) non-parametric approach, which also revealed a significant difference in beta diversity between brushing frequencies $\left(\mathrm{R}^{2}=0.281, p=0.002\right.$, 999 permutations). More specifically, children brushing twice daily had significantly decreased prevalence of Actinobacteria $(p=0.001)$ and significantly increased prevalence of Proteobacteria ( $p=0.025$ ) compared to children brushing once daily, as shown in Figure 1a. The other main phyla were not affected by the tooth brushing frequency.

\section{Discussion}

Findings from this study supported the hypothesis that children's tooth brushing frequency is associated with oral microbiota composition. In our study, the relative abundance of the oral microbiota community changed when tooth brushing frequency increased from once to twice a day (Figure 1b), and children brushing twice a day have a greater relative abundance of Proteobacteria (greater than 35\%) in comparison to Actinobacteria (greater than $10 \%$ ). Interestingly, the difference in oral microbiome in people living in a different place had similarities to our findings in urban areas. A study in Myanmar with supragingival plate samples collected from children aged 9-13 years old living in remote mountains areas 
found that Proteobacteria (58.1\%) account for the highest abundance, followed by Firmicutes $(21.5 \%)$, and Bacteroidetes (14.8\%) [23]. Jiang and colleagues reported similar findings, suggesting that children with abundant Proteobacteria (greater than 50\%) and Actinobacteria (greater than 25\%) developed fewer caries [24]. A recent literature review points out that in North America, a multitude of Proteobacteria and Fusobacteria is observed in children with no caries belonging to the indigenous population [25]. While the mechanism underlying this association remains unexplained, the lower relative abundance of Actinobacteria in children brushing twice a day compared to those brushing once a day may be a reflection of the lower abundance of the genus Actinomyces, which is commonly known as an opportunistic pathogen in the oral cavity. Similarly, while the increased relative abundance of Proteobacteria in children brushing twice a day could be related to the relative decrease in the abundance of Actinobacteria, it would be worth asking if the relative proportions illustrated in the group brushing twice a day might represent a "healthier" composition and abundance in which the commensal microbes keep the cariogenic bacteria at bay.

Contrary to our findings, a study of Irish children who provided saliva samples reported finding Proteobacteria as the dominating caries-active salivary microbiota (median abundance value $38.2 \%$ ) [26]. Jiang and colleagues reported non-significant differences for the alpha and beta diversity in aging adults with or without caries [27]. Similarly, $\mathrm{Xu}$ and colleagues studied the oral microbiota in samples of supragingival plaque and saliva collected from caries and caries-free groups of Chinese children aged 6-8. An analysis of the $16 \mathrm{~S}$ hypervariable region of $\mathrm{V} 1-\mathrm{V} 3$ showed that the overall abundance and diversity of the top 18 phyla varied between the caries and caries-free groups [28]. A study that looked into oral microbiota and caries in monozygotic twins (MZ) and dizygotic twins (DZ) used a methodology with sequencing in the same $16 \mathrm{~S}$ V3-V4 region, but with bioinformatics analysis different from our study [29]. Although we cannot confirm the same results, we do see a microbial similarity with the top five phyla, which corresponds with $97.3 \%$ of the bacteria abundance in their study sample [29].

While the increased relative abundance of Proteobacteria may reflect a pathogenic environment, and the decreased relative abundance of Actinobacteria may indicate microbial imbalances, it is important to note that there is a variation in sampling techniques, population-age group, gene-sequencing platforms, or bioinformatics analysis across studies. In general, our study notes that the diversity and abundance of the oral microbiota affected by tooth brushing once or twice daily may help explain the mechanisms by which tooth brushing helps prevent caries in children. Further analyses using tooth brushing and lower taxonomic levels are warranted.

Our results suggest that each participant's oral microbiota was unique, demonstrating agreement with the idea of commensal diversity within a person that is characterized by tooth brushing frequency. The samples collected from the upper and lower teeth revealed a unique microbial profile in the oral cavity according to the participants' oral health behavior. Each individual has a unique oral microbiome from birth, which resides in an open cavity exposed to the external environment [30]. This external exposure allows the oral microbiota to diversify and adapt to the different environments [30]. Tooth brushing frequency may affect the environmental balance in which the commensal bacteria could thrive. It is worth noting that the presence of specific phyla alone may not predict the development of dental caries, and that other risk factors affecting caries development should be considered in combination with microbiota composition.

The current understanding of dental caries involves multiple risk factors, including sugar intake and socioeconomic status. Some studies have found that children's ethnicity may be associated with changes in oral microbiota composition over time due to environmental exposures in lower socioeconomic and disadvantaged communities [31]. While a breakdown of the microbial profile by ethnicity or socioeconomic status and environmental exposures was not the focus of our study, future studies investigating the influences of sugar intake, acculturalization, as well as parents' oral health behavior and oral microbiome 
on children's microbial profiles may be helpful when designing interventions to optimize oral microbial composition.

A chief limitation in our pilot study was under-representation of female participants, which precluded us from having an accurate representation of the US population. Additionally, a larger sample in which the oral microbiome could be correlated with oral symptoms would be very helpful. With our limited funding, we focused on analyzing microbial composition; expanding the oral microbiome analysis using a combination of metagenomic, metatrascriptomic, and metabolomic analysis is warranted to help us better understand the metabolic profile and activity of the bacteria. In future studies, collecting more information such as types of toothpaste used, dietary patterns such as regular consumption of sugary drinks or processed food, and the oral $\mathrm{pH}$ level would be helpful in determining the moderating role of the oral environment on the microbial profile.

\section{Conclusions}

Tooth brushing frequency can affect the proportional composition of oral microbiota, the composition of which becomes a risk factor for dental caries if not in a "healthy balance." The ultimate goal is to use tooth brushing frequency to change the composition of the oral microbiome for a beneficial balance toward a healthy mouth and beyond. For oral research, this study offers preliminary baseline data showing the association between relative abundance of oral microbiota and tooth brushing frequency. The interdisciplinary approach used in this study, combining clinical practice and basic science research, will help translate research findings to clinical practices that can positively impact children by keeping them healthy in our communities. The development of innovative tooth brushing interventions aimed at promoting tooth brushing behavior in children to optimize the oral microbiota may decrease the risk of both oral and systemic diseases.

Author Contributions: S.J.C., lead principal investigator, performed overall direction, designed the study, obtained the funding, collected oral samples and data, performed DNA extraction and preparation of the oral samples for sequencing, analyzed the data, and drafted the manuscript. S.Y.C., co-principal investigator; assisted in designing the study, collecting oral samples, and editing the manuscript. C.J.F., performed the genomic sequencing and wrote the oral microbiome methods. N.T.M., performed experiments, analyzed data, and wrote sections of the analysis in the manuscript. All authors have read and agreed to the published version of the manuscript.

Funding: This study was funded by Sigma Theta Tau International Xi Pi Chapter Small Grants \#13831, Illinois State University, Mennonite College of Nursing Internal Grant, and supported by America's Promise School Project.

Institutional Review Board Statement: The study was conducted according to the guidelines of the Declaration of Helsinki, and approved by the Institutional Review Board of Illinois State University Institutional Review Board (protocol code IRB-2018-45 and approval day on 30 March 2018).

Informed Consent Statement: Informed consent was obtained from all subjects involved in the study.

Data Availability Statement: The data that support the findings of this study are available from the Illinois State University. Data are available from the author with the permission of the Illinois State University.

Conflicts of Interest: The authors declare no conflict of interest.

\section{References}

1. Lifshitz, F.; Casavalle, P.L.; Bordoni, N.; Rodriguez, P.N.; Friedman, S.M. Oral Health in Children with Obesity or Diabetes Mellitus. Pediatr. Endocrinol. Rev. 2016, 14, 159-167. [CrossRef]

2. Centers for Disease Control and Prevention. QuickStats: Prevalence of Untreated Dental Cariesin Permanent Teeth Among Children and Adolescents Aged 6-19 Years, by Age Group-National Health and Nutrition Examination Survey, United States, 2011-2014, Morbidity and Mortality Weekly Report (MMWR); Centers for Disease Control and Prevention: Atlanta, GA, USA, 2017; Volume 66. [CrossRef]

3. Surgeon General's Report on Oral Health in America I Data \& Statistics I National Institute of Dental and Craniofacial Research. Available online: https:/ / www.nidcr.nih.gov/research/data-statistics/surgeon-general (accessed on 7 April 2020). 
4. Kanellis, M.J.; Damiano, P.C.; Momany, E.T. Medicaid Costs Associated with the Hospitalization of Young Children for Restorative Dental Treatment under General Anesthesia. J. Public Health Dent. 2000, 60, 28-32. [CrossRef]

5. Avenetti, D.; Lee, H.H.; Pugach, O.; Rosales, G.; Sandoval, A.; Martin, M. Tooth Brushing Behaviors and Fluoridated Toothpaste Use among Children Younger than Three Years Old in Chicago. J. Dent. Child. Chic. Ill 2020, 87, 31-38.

6. Wang, B.; Yao, M.; Lv, L.; Ling, Z.; Li, L. The Human Microbiota in Health and Disease. Engineering 2017, 3, 71-82. [CrossRef]

7. Gomez, A.; Nelson, K.E. The Oral Microbiome of Children: Development, Disease, and Implications Beyond Oral Health. Microb. Ecol. 2017, 73, 492-503. [CrossRef]

8. Banas, J.A.; Zhu, M.; Dawson, D.V.; Blanchette, D.R.; Drake, D.R.; Gu, H.; Frost, R.; McCaulley, G.; Levy, S.M. Acidogenicity and Acid Tolerance of Streptococcus Oralis and Streptococcus Mitis Isolated from Plaque of Healthy and Incipient Caries Teeth. J. Oral Microbiol. 2016, 8, 32940. [CrossRef] [PubMed]

9. Lif Holgerson, P.; Öhman, C.; Rönnlund, A.; Johansson, I. Maturation of Oral Microbiota in Children with or without Dental Caries. PLoS ONE 2015, 10, e0128534. [CrossRef] [PubMed]

10. Shang, Q.; Gao, Y.; Qin, T.; Wang, S.; Shi, Y.; Chen, T. Interaction of Oral and Toothbrush Microbiota Affects Oral Cavity Health. Front. Cell. Infect. Microbiol. 2020, 10, 17. [CrossRef]

11. Lu, N.; Han, Y.; Chen, T.; Gunzler, D.D.; Xia, Y.; Lin, J.Y.; Tu, X.M. Power Analysis for Cross-Sectional and Longitudinal Study Designs. Shanghai Arch. Psychiatry 2013, 25, 259-262. [CrossRef]

12. Watts, G.S.; Youens-Clark, K.; Slepian, M.J.; Wolk, D.M.; Oshiro, M.M.; Metzger, G.S.; Dhingra, D.; Cranmer, L.D.; Hurwitz, B.L. 16S RRNA Gene Sequencing on a Benchtop Sequencer: Accuracy for Identification of Clinically Important Bacteria. J. Appl. Microbiol. 2017, 123, 1584-1596. [CrossRef]

13. Behjati, S.; Tarpey, P.S. What Is next Generation Sequencing? Arch. Dis. Child. Educ. Pract. Ed. 2013, 98, 236-238. [CrossRef]

14. Oksanen, J.; Blanchet, F.G.; Friendly, M.; Kindt, R.; Legendre, P.; McGlinn, D.; Minchin, P.R.; O’Hara, R.B.; Simpson, G.L.; Solymos, P.; et al. Vegan: Community Ecology Package. Available online: https:/ /CRAN.R-project.org/package=vegan (accessed on 28 August 2018).

15. R Core Team. R: A Language and Environment for Statistical Computing; R Foundation for Statistical Computing: Vienna, Austria, 2019.

16. Quast, C.; Pruesse, E.; Yilmaz, P.; Gerken, J.; Schweer, T.; Yarza, P.; Peplies, J.; Glockner, F.O. The SILVA Ribosomal RNA Gene Database Project: Improved Data Processing and Web-Based Tools. Nucleic Acids Res. 2013, 41, D590-D596. [CrossRef]

17. Wright, E.S. DECIPHER: Harnessing Local Sequence Context to Improve Protein Multiple Sequence Alignment. BMC Bioinform. 2015, 16, 322. [CrossRef]

18. Price, M.N.; Dehal, P.S.; Arkin, A.P. FastTree 2-Approximately Maximum-Likelihood Trees for Large Alignments. PLoS ONE 2010, 5, e9490. [CrossRef] [PubMed]

19. Lan, Y.; Wang, Q.; Cole, J.R.; Rosen, G.L. Using the RDP Classifier to Predict Taxonomic Novelty and Reduce the Search Space for Finding Novel Organisms. PLoS ONE 2012, 7, e32491. [CrossRef]

20. McMurdie, P.J.; Holmes, S. Phyloseq: An R Package for Reproducible Interactive Analysis and Graphics of Microbiome Census Data. PLoS ONE 2013, 8, e61217. [CrossRef]

21. Davis, N.M.; Proctor, D.M.; Holmes, S.P.; Relman, D.A.; Callahan, B.J. Simple Statistical Identification and Removal of Contaminant Sequences in Marker-Gene and Metagenomics Data. Microbiome 2018, 6, 226. [CrossRef]

22. Pinheiro, J.; Bates, D.; DebRoy, S.; Sarkar, D.; Team, R.C. Nlme: Linear and Nonlinear Mixed Effects Models; R Package Version 3.1-103. Available online: https:/ /CRAN.R-project.org/package=nlme (accessed on 28 August 2018).

23. Nomura, Y.; Otsuka, R.; Hasegawa, R.; Hanada, N. Oral Microbiome of Children Living in an Isolated Area in Myanmar. Int. J. Environ. Res. Public Health 2020, 17, 4033. [CrossRef] [PubMed]

24. Jiang, S.; Gao, X.; Jin, L.; Lo, E.C.M. Salivary Microbiome Diversity in Caries-Free and Caries-Affected Children. Int. J. Mol. Sci. 2016, 17, 1978. [CrossRef]

25. Fakhruddin, K.S.; Ngo, H.C.; Samaranayake, L.P. Cariogenic Microbiome and Microbiota of the Early Primary Dentition: A Contemporary Overview. Oral Dis. 2019, 25, 982-995. [CrossRef] [PubMed]

26. Hurley, E.; Barrett, M.P.J.; Kinirons, M.; Whelton, H.; Ryan, C.A.; Stanton, C.; Harris, H.M.B.; O’Toole, P.W. Comparison of the Salivary and Dentinal Microbiome of Children with Severe-Early Childhood Caries to the Salivary Microbiome of Caries-Free Children. BMC Oral Health 2019, 19, 13. [CrossRef]

27. Jiang, Q.; Liu, J.; Chen, L.; Gan, N.; Yang, D. The Oral Microbiome in the Elderly with Dental Caries and Health. Front. Cell. Infect. Microbiol. 2019, 8, 442. [CrossRef]

28. Xu, Y.; Jia, Y.H.; Chen, L.; Huang, W.M.; Yang, D.Q. Metagenomic Analysis of Oral Microbiome in Young Children Aged 6-8 Years Living in a Rural Isolated Chinese Province. Oral Dis. 2018, 24, 1115-1125. [CrossRef] [PubMed]

29. Kasimoglu, Y.; Koruyucu, M.; Birant, S.; Karacan, I.; Topcuoglu, N.; Tuna, E.B.; Gencay, K.; Seymen, F. Oral Microbiota and Dental Caries Data from Monozygotic and Dizygotic Twin Children. Sci. Data 2020, 7, 348. [CrossRef] [PubMed]

30. Dzidic, M.; Collado, M.C.; Abrahamsson, T.; Artacho, A.; Stensson, M.; Jenmalm, M.C.; Mira, A. Oral Microbiome Development during Childhood: An Ecological Succession Influenced by Postnatal Factors and Associated with Tooth Decay. ISME J. 2018, 12, 2292-2306. [CrossRef] [PubMed]

31. Soncini, J.A.; Kanasi, E.; Lu, S.C.; Nunn, M.E.; Henshaw, M.M.; Tanner, A.C.R. Oral Microbiota of Children in a School-Based Dental Clinic. Anaerobe 2010, 16, 278-282. [CrossRef] 